\title{
Multi-Agent Robot System and Events Executing Using GPS Cloud
}

\author{
Abdellah Redjaimia ${ }^{1, *}$, Ghomrani Mohammed El Amine ${ }^{2}$ \\ ${ }^{1}$ Department of Mathematics and Informatics Lobachevsky State University of Nizhni Novgorod, Nizhni Novgorod, Russia \\ ${ }^{2}$ Department of Economics and Entrepreneurship - Mathematical and tool methods of economy Lobachevsky State University of Nizhni \\ Novgorod, Nizhni Novgorod, Russia
}

Email address:

redjaimia@gmail.com (A. Redjaimia),ghomranipeter89@gmail.com (G. M. El Amine)

To cite this article:

Abdellah Redjaimia, Ghomrani Mohammed El Amine. Multi-agent Robot System and Events Executing Using GPS Cloud. American Journal of Remote Sensing. Vol. 4, No. 1, 2016, pp. 1-8. doi: 10.11648/j.ajrs.20160401.11

\begin{abstract}
Large number of autonomous robot solutions exists for various missions and domains. These robots are sufficient for the missions they are built for. At the same time each of them has limited functional and physical capabilities. Multi agent systems can be used to remove these limits. However it is true only in case when the system ensures effective interaction among the robots i.e. enables their social behavior. Usually it is hard to implement such capabilities directly into robots due to functional and physical limitations and heterogeneity of the team. One of possible solutions is to implement a behavior sensors management for the robots. [1] It should collect events, allocate subtasks to specific robots and monitor the execution of the assigned tasks. In order to avoid inherent drawback of fully centralized systems a significant level of autonomy has to be preserved. Intelligent agents fulfill these requirements. Therefore we propose a multi-agent system's architecture for safe road application with GPS tool. It can be used to control the car speed and to adjust it in case of danger.
\end{abstract}

Keywords: Multi-Agent, Agent, GPS, Speed Limit Value, Car Speed Value, Thread, Road Speed Board, Speed Sensor

\section{Introduction}

Various robots for different purposes exist. Autonomous vacuum cleaning robots clean various premises, agricultural robots do many jobs in precise agriculture, etc. Usually these robots include algorithms for autonomous execution of their missions. [1] For example, a vacuum cleaning robot is intelligent enough to autonomously clean a certain area in sufficient quality. Unfortunately these robots still have significant limitations. For instance, a single robot cannot be effectively used for larger (more efforts requiring) missions, where it is not capable to accomplish the whole mission [1]. Example of such mission is a large area that cannot be cleaned by a single vacuum cleaning robot because of time and resource considerations. One of possible solutions is to use multiple robots simultaneously for a given mission.

In this paper we discuss a multi-agent system which can be plugged inside a car as a terminal and execute tasks via a group of agents which they collect events through sensors such as: wind, rain, temperature, speed... etc.

we focus on one of those events which is the main one cause of its necessary on road phenomenon and cause of it a lot of people are losing their lives daily [2] [3] [4]. We try to explain how via a GPS cloud a plugged robot in car can force the driver to respect the Speed limit which can be taken as a parameter from a GPS sensor.

\section{Statistics}

This list of countries by traffic-related death rate shows the annual number of road fatalities per capita per year and per vehicle- $\mathrm{km}$ in some countries in the year the data was collected [2].

According to the World Health Organization, road traffic injuries caused an estimated 1.24 million deaths worldwide in the year 2010, slightly down from 1.26 million in 2000 . That is one person is killed every 25 seconds. Only 28 countries, representing 449 million people ( $7 \%$ of the world's population), have adequate laws that address all five risk factors (speed, drink-driving, helmets, seat-belts and child restraints). Over a third of road traffic deaths in low- and middle-income countries are among pedestrians and cyclists. However, less than $35 \%$ of low- and middle-income countries have policies in place to protect these road users. 
The average rate was 18 per 100,000 people (down from 20.8 in 2000). However, middle-income countries have the highest annual road traffic fatality rates, at 20.1 per 100000 , while the rate in high-income countries is lowest, at 8.7 per 100000 .

Eighty per cent of road traffic deaths occur in middleincome countries, which account for $72 \%$ of the world's population, but only $52 \%$ of the world's registered vehicles. This indicates that these countries bear a disproportionately high burden of road traffic deaths relative to their level of motorization [2].

There are large disparities in road traffic death rates between regions. The risk of dying as a result of a road traffic injury is highest in the African Region (24.1 per 100000 population), and lowest in the European Region (10.3 per $100000)$ [2].

Half of the world's road traffic deaths occur among motorcyclists $(23 \%)$, pedestrians $(22 \%)$ and cyclists $(5 \%)-$ i.e. "vulnerable road users" - with $31 \%$ of deaths among car occupants and the remaining 19\% among unspecified road users.

Adults aged between 15 and 44 years account for $59 \%$ of global road traffic deaths. $77 \%$ road deaths are among men.

The total fatalities figures comes from the WHO report (table A2, column point estimate, pp. 242-255) and are often an adjusted number of road traffic fatalities in order to reflect the different reporting and counting methods among the many countries (e.g. "a death after how many days since accident event is still counted as a road fatality?" (by standard adjusted to a 30 days period), or "to compensate for underreporting in some countries", see WHO report pp. 48-51).

High speed reduces the possibility to respond in time when necessary. People need time to process information, to decide whether or not to react and, finally to execute a reaction. At high speed the distance covered in this period is longer. At high speeds the distance between starting to brake and a complete stand still is longer as well. The braking distance is proportional to the square of speed (v2). Therefore, the possibility to avoid a collision becomes smaller as speed increases. This is well illustrated at a broad average level by Finch.

$1 \mathrm{~km} / \mathrm{h}$ increase in speed $\rightarrow 3 \%$ increase in accidents [2].

In practice the relationship is more complex. The exact relationship depends among many other things on speed level and road type.

The higher the speed, the steeper the increase in accident risk [2].

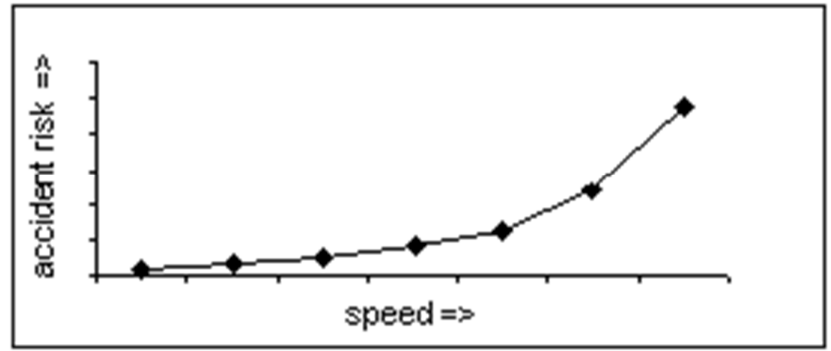

Figure 1. Graph explains the relation between Speed and accident risk [2].
The relationship between speed and accident risk is a power function: With increasing speed, the accident risk increases more as the absolute speed is higher [2].

Based on the principles of kinetic energy and validated by empirical data, Nilsson developed the following formula:

$$
A_{2}=A_{1}\left(\frac{v_{2}}{v_{1}}\right)^{2}
$$

In words: the number of injury accidents after the change in speed (A2) equals the number of accidents before the change (A1) multiplied by the new average speed (v2) divided by the former average speed (v1), raised to the square power.

Also road type affects the relationship speed-accident risk

On some roads the traffic situation is more complex than on other roads. This depends for example on the number and type of intersections; the absence or presence of pedestrians, cyclists, agricultural vehicles. In more complex traffic situations, the accident risk is higher. In addition, the increase of accident risk is larger as complexity increases Taylor. An example of a low complexity road type is a motorway. An example of a high complexity road type is an urban arterial road.

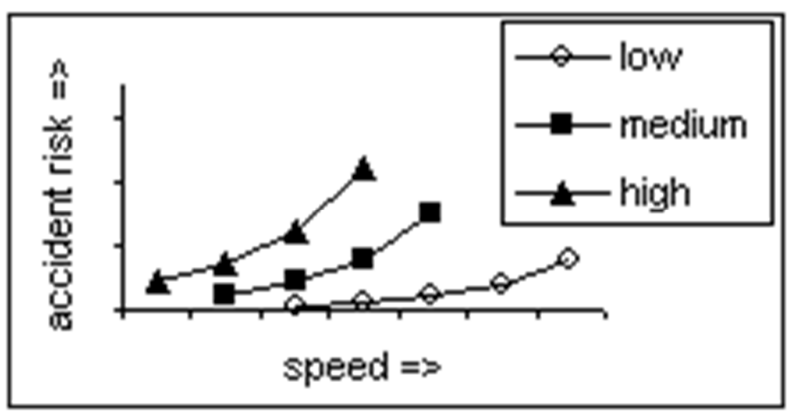

Figure 2. Graph explains the relation between Speed and accident risk -case traffic situation.

\section{Annual Global Road Crash Statistics}

- Nearly 1.3 million people die in road crashes each year, on average 3,287 deaths a day.

- An additional 20-50 million are injured or disabled.

- More than half of all road traffic deaths occur among young adults ages 15-44.

- Road traffic crashes rank as the 9th leading cause of death and account for $2.2 \%$ of all deaths globally.

- Road crashes are the leading cause of death among young people ages 15-29, and the second leading cause of death worldwide among young people ages 5-14.

- Each year nearly 400,000 people under 25 die on the world's roads, on average over 1,000 a day.

- Over $90 \%$ of all road fatalities occur in low and middleincome countries, which have less than half of the world's vehicles.

- Road crashes cost USD \$518 billion globally, costing individual countries from $1-2 \%$ of their annual GDP.

- Road crashes cost low and middle-income countries 
USD \$65 billion annually, exceeding the total amount received in developmental assistance.

- Unless action is taken, road traffic injuries are predicted to become the fifth leading cause of death by 2030 .

\section{GPS}

The GPS is the U.S. Global Navigation Satellite System (GNSS) which provides free positioning and timing services worldwide. GPS receivers compute their position in the GPS Reference System using satellite technology and based on triangulation principles (please refer to GNSS positioning). Originally developed for the U.S. military, the incident with the Korean Air Lines Flight 007 led the US Government to decide to make GPS use free for civilian purposes very early in the experimental phase of GPS [5]. The launch of the first Block I Navistar GPS satellite meant the beginning of the deployment of the GPS system on 22 February 1978, followed by the declaration of the Initial Operating Capability in December 1993 with 24 operational satellites in orbit, and the Full Operational Capability in June 1995. GPS is maintained by the United States government and is freely accessible by anyone with a GPS receiver. The Department of Defense is responsible for operating the system, but it also receives national-level attention and guidance through the National Executive Committee for Space-Based Positioning, Navigation, and Timing (PNT).

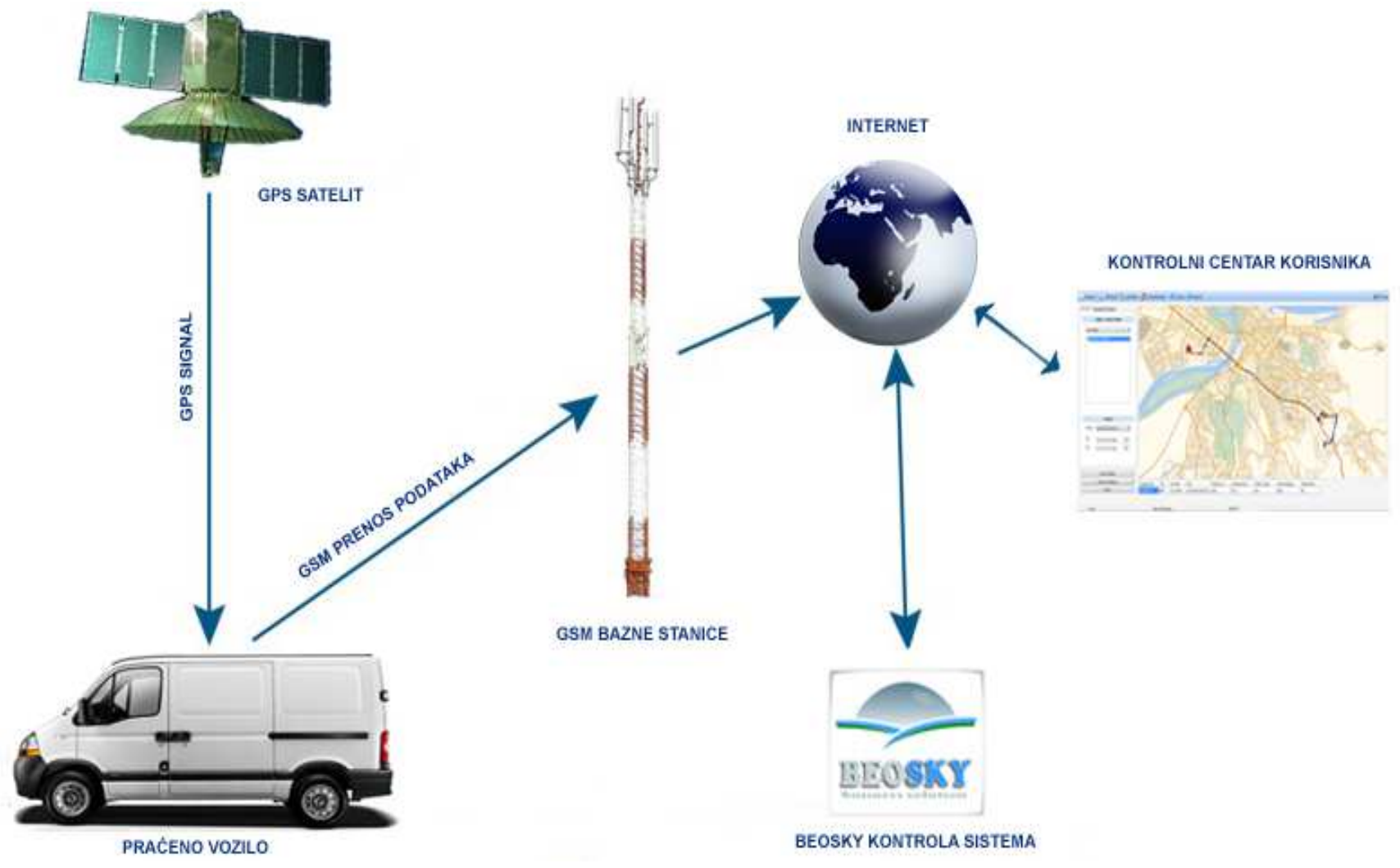

Figure 3. GPS Schema.

Nowadays GPS cloud has plenty features such as road map detection, road traffic signalization and speed limit displayer. all those features have a big value to give the right information to the driver to control his/her car during driving but even GPS is a useful tool which can guarantee a safe road for its users we read every moment in newspapers or we watch daily the road crimes and that it is a big prove which shows us that people (Car Drivers) don't respect plenty tools which they are using and statistics are not believable basing on the technology plugging for each user. The question how can we decrease the road crashes (Accidents) if the user (Drivers) don't care on their lives and their passengers which are getting a sit with them during driving trip? Our research is basing on this question how to guarantee a safe trip during driving?

\subsection{GPS Speed Detection}

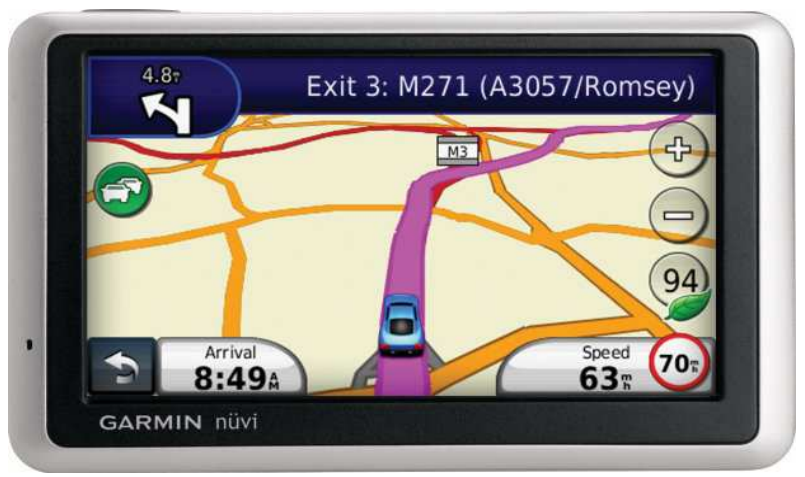

Figure 4. GPS road detection. 
With the advance of handheld GPS (Global Positioning System) devices speed measurements for example of speed surfers or speed kiters become affordable for everyone. GPS units receive signals at a frequency of $1575.42 \mathrm{Mhz}$ from typically up to twelve of the 28 satellites orbiting the Earth in about $20.180 \mathrm{~km}$ height. From the time differences of the satellite signals the distances to the satellites and finally the unit's position (latitude, longitude, and altitude) is calculated [6].

\subsection{Intelligent Speed Adaptation (GPS Speed Limit Detection)}

Intelligent Adaptation (ISA), also known as, Alerting, and Intelligent Authority, is any system that vehicle speed and the local speed limit on a road and implements an action when the vehicle is detected to be exceeding the speed limit. This can be done through an advisory system, where the driver is warned, or through an intervention system where the driving systems of the vehicle are controlled automatically to reduce the vehicle's speed [6].

Intelligent speed adaptation uses information about the road on which the vehicle travels to make decisions about what the correct speed should be. This information can be obtained through use of a incorporating roadway coordinates as well as data on the, through general speed zoning information for a defined geographical area (e.g., an urban area which has a single defined speed limit), or through feature recognition technology that detects and interprets speed limit signage. ISA systems are designed to detect and alert a driver when a vehicle has entered a new speed zone, when variable speed zones are in force (e.g., variable speed limits in school zones that apply at certain times of the day and only on certain days), and when temporary speed zones are imposed (such as speed limit changes in adverse weather or during traffic congestion, at accident scenes, or near road works). Many ISA systems will also provide information about locations where hazards may occur (e.g., in high pedestrian movement areas, railway level crossings or railroad grade crossings, schools, hospitals, etc.) or where enforcement actions is indicated (e.g., speed camera and red light camera locations). The purpose of ISA is to assist the driver in keeping to the lawful speed limit at all times, particularly as they pass through different speed 'zones'. This is particularly useful when drivers are in unfamiliar areas or when they pass through areas where variable speed limits are used.

Research [citation needed] has found that that, in urban areas, the risk of a casualty crash is doubled for each $5 \mathrm{~km} / \mathrm{h}$ over the limit. So travelling at $70 \mathrm{~km} / \mathrm{h}$ in a $60 \mathrm{~km} / \mathrm{h}$ zone quadruples the risk of a crash in which someone is hospitalized. As a result, it is estimated that about $10 \%$ of casualties could be prevented if the large group of motorists who routinely travel at up to $10 \mathrm{~km} / \mathrm{h}$ over the limit were encouraged to obey the speed limits. About $20 \%$ of casualties could be prevented if all vehicles complied with the speed limits. Savings in fatal crashes would be larger [7].

"Minor" speeding therefore makes up a large proportion of preventable road trauma. It is difficult for enforcement methods alone to have an effect on this minor speeding. An added problem is that even motorists who want to obey the speed limits (to keep their life, license or livelihood) have difficulty doing so in modern cars on city roads. This is where an ISA system comes into its own

$N B$ : Speed limit is the speed which is displayed on roads and the one that the driver can't across it, the speed limit changes from a country to another and from city to another too in the same country.

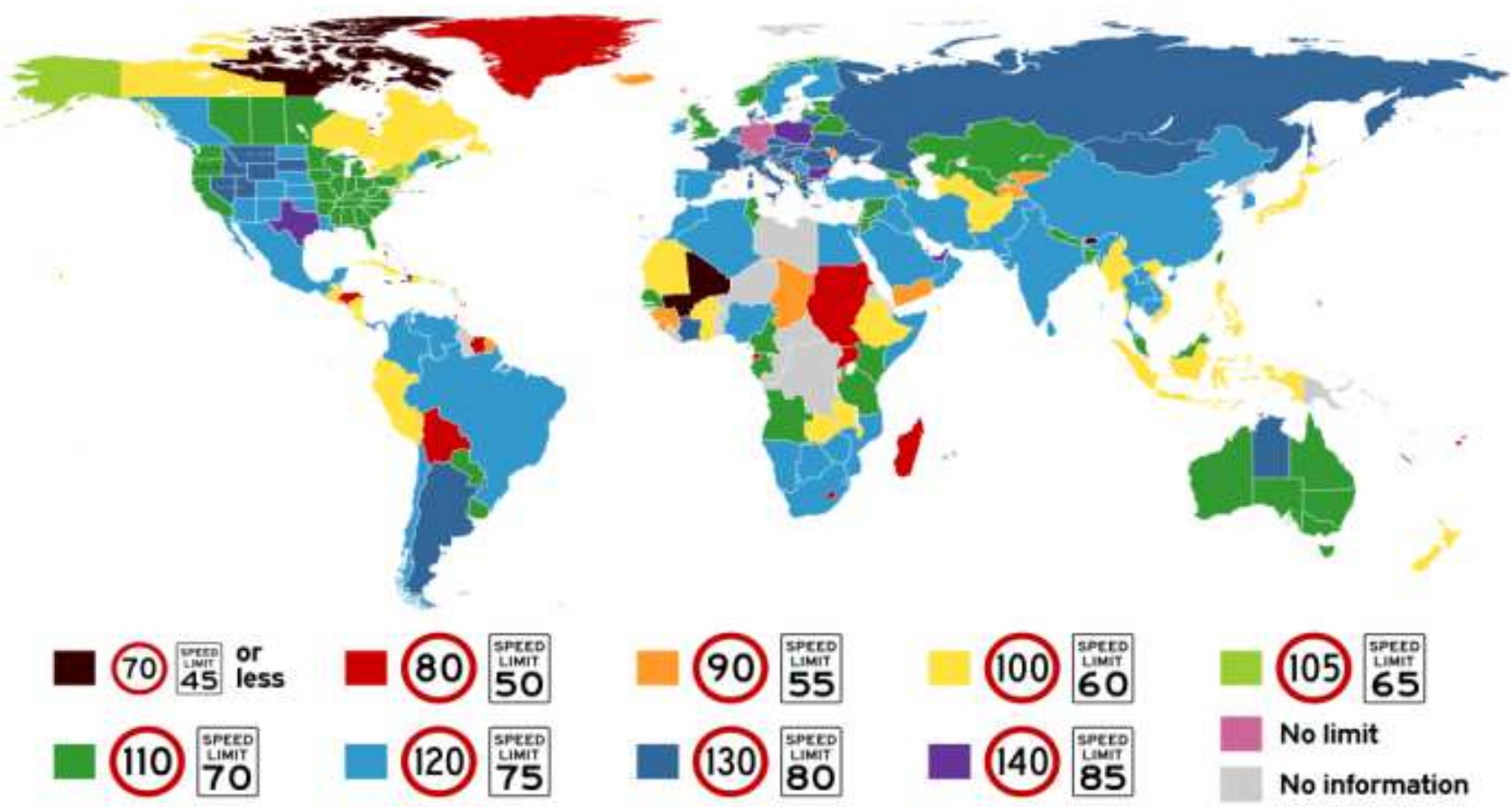

Figure 5. Speed Limit Values depending on countries [6] [7]. 


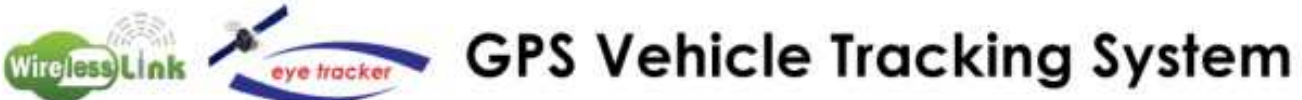

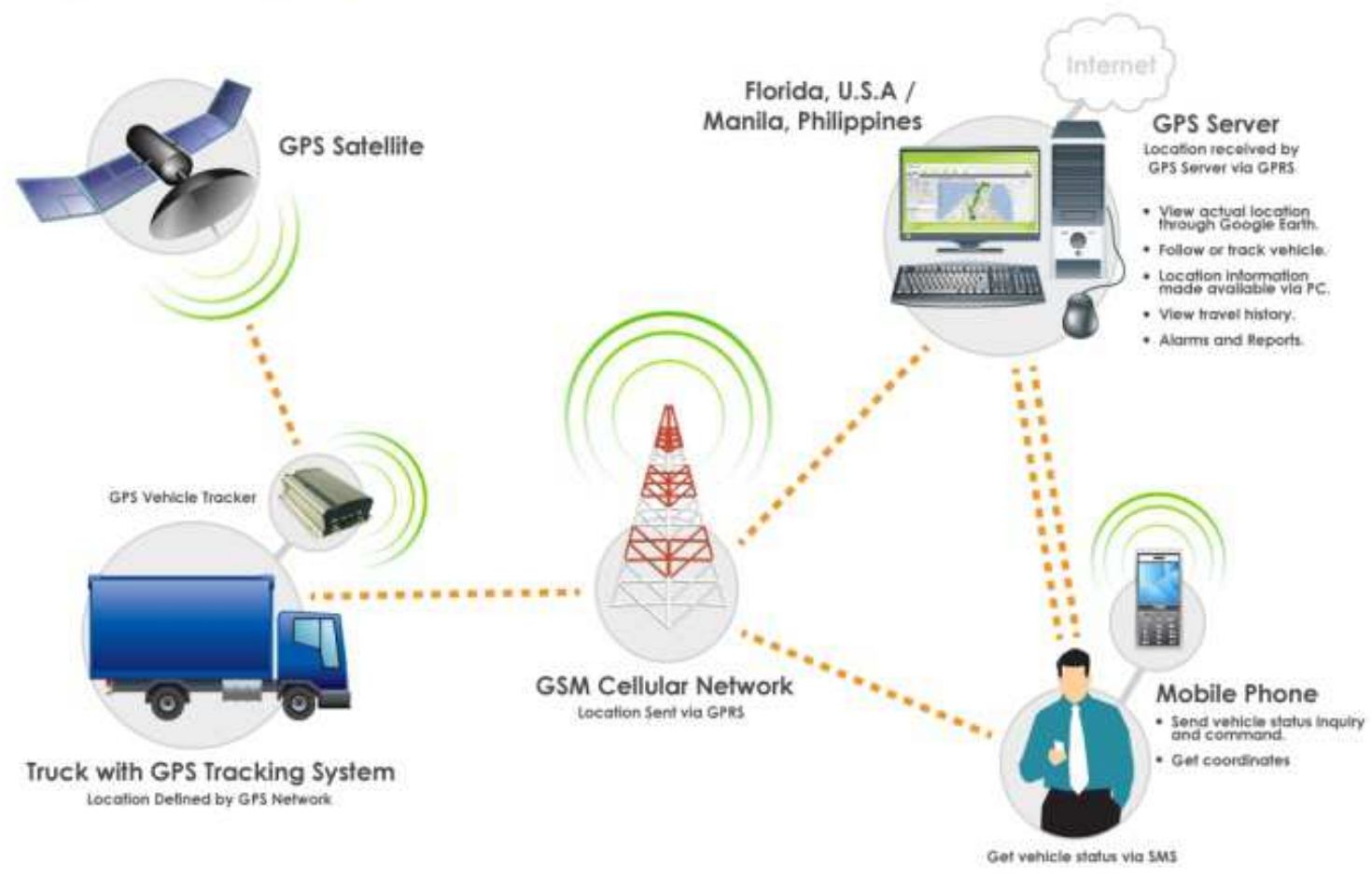

Figure 6. GPS Tracking System.

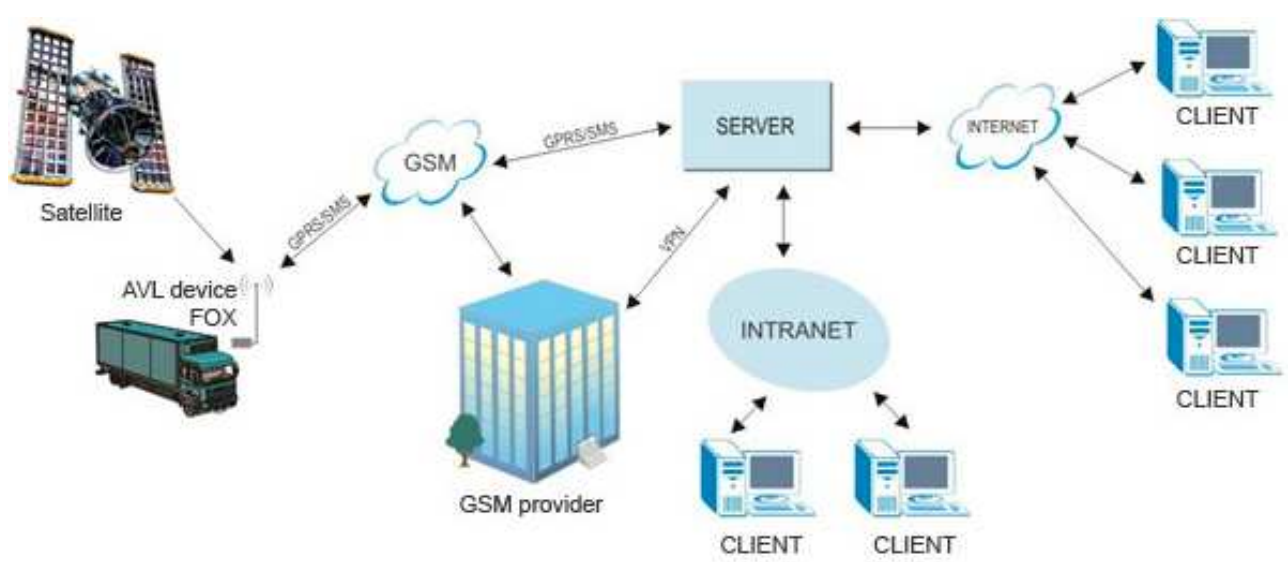

Figure 7. GPS Data Storing.
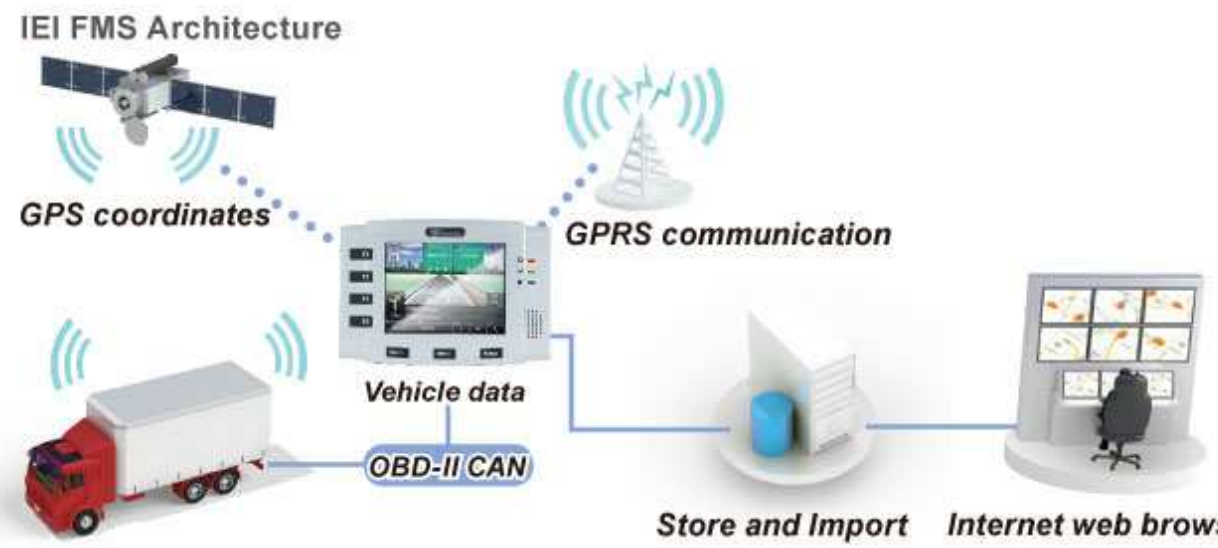

Store and Import Internet web browser

Figure 8. GPS Local Data Storing. 
Nowadays GPS has the ability to detect the speed limit basing on the map and traffic signs which are charged inside the circuit (Satellite Collecting Data) then it is easy for GPS to display more information such as to warn the driver that the speed is limited and you can't pass it or to show the way how to reach a place using the best suggestions.

\section{Multi Agent System and Safe Road Instructions Basing on Speed Limit Value}

in this section we want to show and to make some experiments on the Multi-Agent System and how can help decreasing the number of car road crashes which are daily happening and as it is known the main cause is speed that is mean the human side which doesn't respect the traffic signs [9]. for that the multi agent system suggests a small plan for the assistant which is plugged already inside the car and basing on GPS speed limit detection value the agent will make some rules if necessary [8]. [10]. [11].

Function Verify Current Speed

The function has to be basing on thread process which will stop it is running just when it will find it is condition turned to a True value [12].

What is a Thread?

In computer science, a thread of execution is the smallest sequence of programmed instructions that can be managed independently by a scheduler, which is typically a part of the operating system.[1] The implementation of threads and processes differs between operating systems, but in most cases a thread is a component of a process. Multiple threads can exist within the same process, executing concurrently (one starting before others finish) and share resources such as memory, while different processes do not share these resources. In particular, the threads of a process share its instructions (executable code) and its context (the values of its variables at any given moment).

On a single processor, multithreading is generally implemented by time slicing (as in multitasking), and the central processing unit (CPU) switches between different software threads. This context switching generally happens frequently enough that the user perceives the threads or tasks as running at the same time (in parallel). On a multiprocessor or multi-core system, multiple threads can be executed in parallel (at the same instant), with every processor or core executing a separate thread simultaneously; on a processor or core with hardware, separate software threads can also be executed concurrently by separate hardware threads.

Threads made an early appearance in OS/360 Multiprogramming with a Variable Number of Tasks (MVT) in 1967, in which they were called "tasks". Process schedulers of many modern operating systems directly support both time-sliced and multiprocessor threading, and the operating system kernel allows programmers to manipulate threads by exposing required functionality through the system call interface. Some threading implementations are called kernel threads, whereas lightweight processes (LWP) are a specific type of kernel thread that share the same state and information. Furthermore, programs can have user-space threads when threading with timers, signals, or other methods to interrupt their own execution, performing a sort of ad hoc time-slicing.

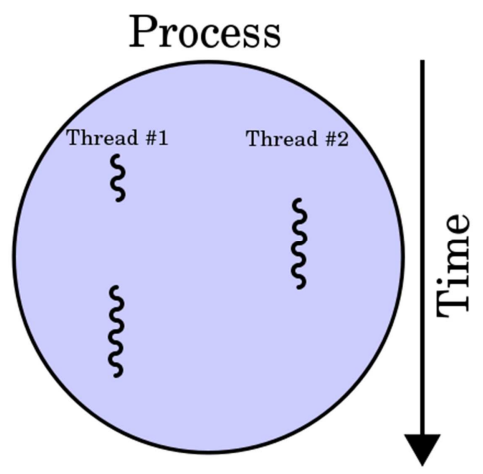

Figure 9. The Thread Mechanism.

The Function Parameters:

The function needs 02 parametres

1- Speed Limit Value.

2- Car Speed Value.

The Speed Limit Value is the speed which is allowed in the current geographic area mostly the value it can be got by a GPS terminal from Road Speed Board which are planted at every way of driving.

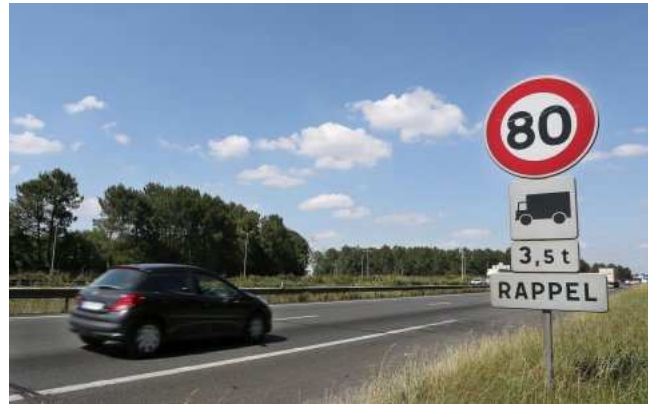

Figure 10. The GPS Speed Limit Boards

The Car Speed Value is the speed which the driver is using it at the moment this speed it can be got also by a GPS terminal or by a Speed Sensor.

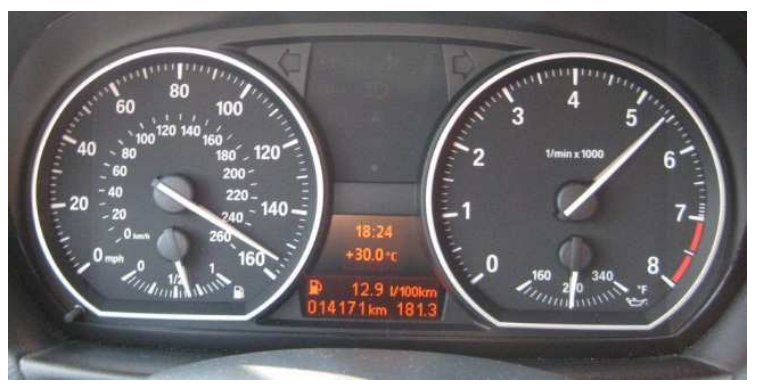

Figure 11. The Speed Sensor. 
Basing on those two parameters we can say that our agents (Car Assistant) will be ready to execute their tasks every time there are changes on both values Speed Limit Value and Car Speed Value.

The Function Structure:

import javax.swing.*;

public class SafeRoadController implements Runnable \{

//Attributes -- Parameters

public static int SpeedLimitValue;

//the SpeedLimitValue is the limit speed

which given by the Robot GPS Sensor

on the current geographic location

basing almost on satellite who got the

value from road speed boards.

public static int CarSpeedValue;

//the CarSpeedValue is the current car

speed which given by the Car Speed

Sensor.

//The Class Constructor

public SafeRoadController() \{\}

//Methods

//getSpeedLimitValue

public int getSpeedLimitValue

(GPS gps) \{

SpeedLimitValue $=$ gps.getLimit () ;

// calling GPS to assist giving the current speed limit displayed on the current geographic position. return SpeedLimitValue; \}

//getCarSpeedValue

public int getCarSpeedValue

(CAR car) \{

CarSpeedValue $=$ car.getSpeed () ;
// calling the Car Speed Sensor to assist giving the current speed used by the Driver. return CarSpeedValue;

//Thread Preparation.

//Main Method plus the Main Process public static void main(String [] args $)\{$ while(true) \{

if(SafeRoadController.SpeedLimitValue $<$ SafeRoadController.CarSpeedValue $)\{$ // small test to detect if the driver is in danger or he/she doesn't respect the regular speed which he/she has to use on that geographic position. JOptionPane.showMessageDialog(new JFrame(), "Your speed is High, Slow Down or the Car will do that automatically after 5 seconds"); CarSpeedValue $=$ SpeedLimitValue; // if the user (Driver) will not slow down the Robot (Assistant) will act and slow down the current car speed to reach the current speed limit.

\}) \}

//End Class $\}$

$N B$ : The source is simulated with JAVA programming language (Object Oriented Language) just to explain the agents mechanism and it is reactions when they receive events (Parameters) in our case events are represented with both attributes: SpeedLimitValue and CarSpeedValue.

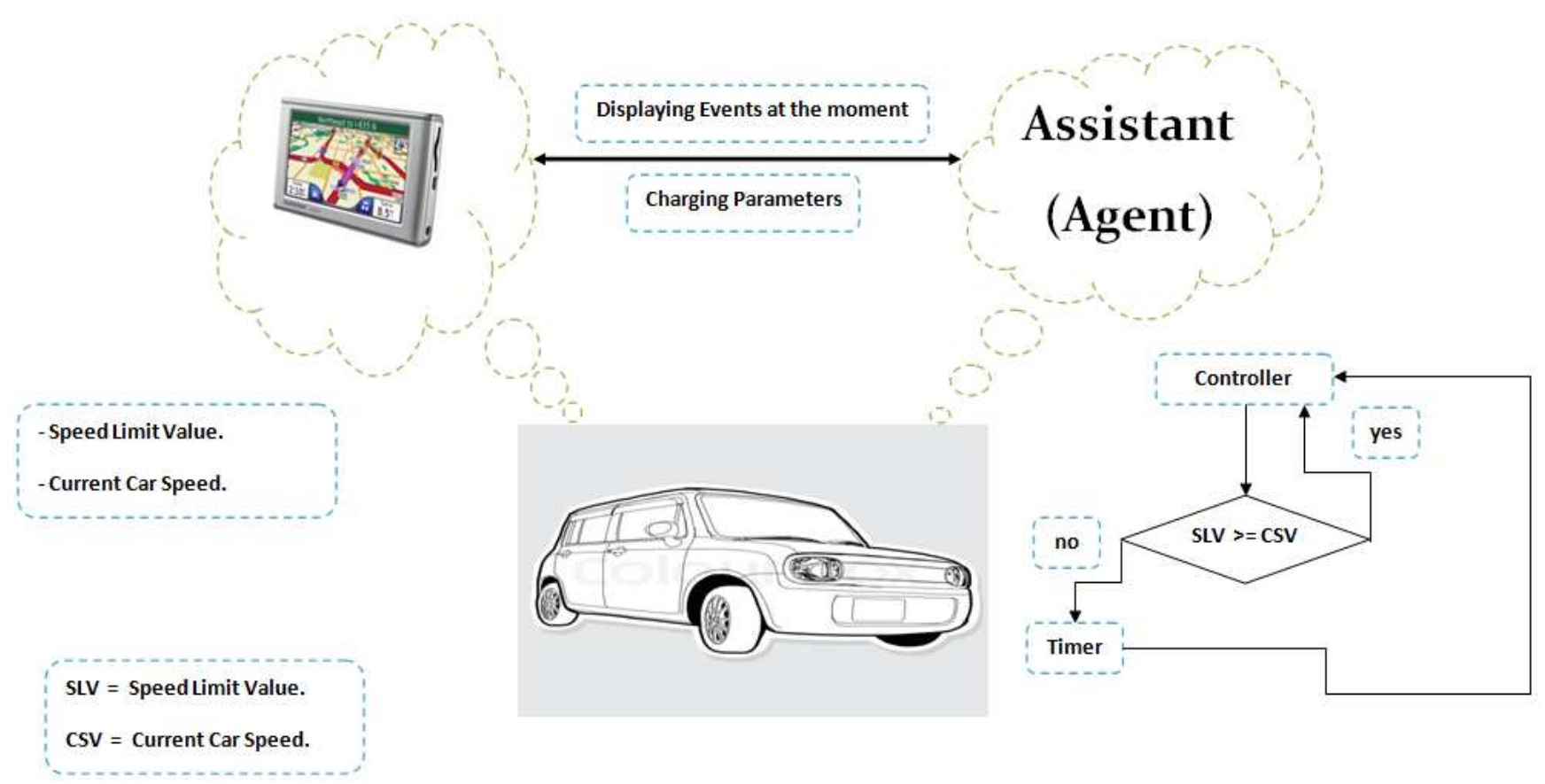

Figure 12. Multi-agent Robot System and Events Executing Using GPS Cloud The System Diagram. 


\section{Conclusion}

Road traffic collisions and incidents often have more than one person to blame but in all cases the main cause is the human be. However, many of the reasons involve the drivers directly, even in cases where they are simply responsible because they were not conscientious of adverse driving conditions (Speed Limit - Traffic Signs... etc).

Many solutions governments tried to apply they could for example be pressed to make intersections safer, or to put barriers on every road to stop people going off road. But, the biggest factor is the driver, and all those tries didn't help or give the results which they were expected.

The idea is how to make the Driver respecting all the road obligations? How to force him/her to slow down when the road speed limit shown on every road entrance? The fact drivers don't care on all those signs and they prefer to sort their problems out as fast as possible without caring on those whom are using their foot to cross roads and sometimes is worse cause even drivers don't care on their passengers.

Multi-Agent Robot System and Events Executing Using GPS Cloud is the system which guarantee that drivers for sure will respect the traffic signs (Speed Limit - Traffic lights... etc) or the system will make them losing the car's control and the system will charge that.

\section{References}

[1] Multi-Agent Robotic System Architecture for Effective Task Allocation and Management Egons Lavendelis, Aleksis Liekna, Agris Nikitenko, Arvids Grabovskis and Janis Grundspenkis Department of Systems Theory and Design Riga Technical University.
[2] Improving the response to road death and injury Road Peace conference (April 2008) by Brigitte Chaudhry MBE, Founder $\&$ President of Road Peace.

[3] Road Traffic Accidents Increase Dramatically Worldwide (March 2006) by Heidi Worley.

[4] ROAD TRAFFIC ACCIDENT: A LEADING CAUSE OF THE GLOBAL BURDEN OF PUBLIC HEALTH INJURIES AND FATALITIES by Shahnewaz Rabbi.

[5] GPS Trajectory Feature Extraction for Driver Risk Profiling by Johannes Paefgen, Florian Michahelles and Thorsten Staake.

[6] Vehicle speed estimation using GPS/RISS (Reduced Inertial Sensor System) by O'Kane, T. and Ringwood, J.V. (June 2013)

[7] AUGMENTING GPS SPEED LIMIT MONITORING WITH ROAD SIDE VISUAL INFORMATION by M.L. Eichner, T.P. Breckon.

[8] Improvement of multisensor fusion in speed limit determination by quantifying navigation reliability by A.- S. Puthon, Fawzi Nashashibi and Benazouz Bradai.

[9] Continuing Research in Multi-Agent Systems by K. Decker, M. Fisher, M. Luck, M. Tennenholtz, and UKMAS Contributorsy.

[10] Progress in Multi-Agent Systems Research by Omer Rana, Chris Preist, Michael Luck (March 2000).

[11] Research and Implementation of Multi- agent Based Test Paper Generation Algorithm by Wang Hairui (December 2008).

[12] Conference Paper Multi-Agent Robotic System Architecture for Effective Task Allocation and Management by Egons Lavendelis, Aleksis Liekna, Agris Nikitenko, Arvids Grabovskis and Janis Grundspenkis. 